\title{
Kinds of Information in Scientific Use
}

\author{
John Collier
}

collierj@ukzn.ac.za, School of Philosophy and Ethics, University of KwaZulu-Natal, Durban, South Africa.

\begin{abstract}
There are many different mathematical definitions of information that have their various uses, but I will be concerned with notions of information used in applications in various branches of science that are distinguished by their topic, i.e., what they apply to. I describe the major uses information, and show their relations to each other. I will argue that the various uses form a nested hierarchy, in which each is a restriction on the previous, inheriting the properties of its predecessor, but adding in new features that make it a special case. The lowest level is physical information determined by distinctions and the highest is explicit representation in linguistic social communication. Is there anything common to information at all these levels? I will argue that there is, and that information in each case is what Donald MacKay (1969) called a distinction that makes a difference. What distinguishes the use of information at each level is what distinctions make a causal difference at that level. At each successive level distinctions that make a difference at a previous level make no difference at that level. In order to create this sort of filter new levels have to be formed by cohesion peculiar to the identifying characteristics at that level. A consequence of this view is that information must have causal powers, and that there is a tight connection between information and causation.
\end{abstract}

Keywords: Information, causation, "it from bit", entropy, natural hierarchy, function, representation, intentionality, meaning, reference, communication

Acknowledgement: Acknowledgement text acknowledgement text acknowledgement text acknowledgement text acknowledgement text acknowledgement text acknowledgement text acknowledgement text acknowledgement text acknowledgement text acknowledgement text acknowledgement text

Information has proven to be an elusive concept. I will argue that this is at least in part because it has a variety of uses in the sciences, and various parties tend to claim that their own use refers to the "real" information. There are other reasons for the elusiveness of the concept, not the least being that there seems to be no single concept that ties various usages of information concepts together. We might therefore conclude, a popular trend these days with diverse concepts, that a pluralistic approach to information is the best that we can do, and even that this reflects not limitations on our abilities, but objective reality. Furthermore there is sometimes confusion between various ways of representing or measuring information and the disparate ways in which information can be said to exist in our world. Information has a number of mathematical definitions that are useful, often in different contexts. It is an interesting question to what degree these definitions can be unified into a single mathematical theory of information. However, this is not my subject here, nor is the issue of how various mathematical definitions of information can be used to represent or measure information as it exists in the world.

I am interested here in what I call substantive information, i.e., information not as it is perceived or measured (e.g., through instruments, or to speak more generally, instrumentally), but as it would make a difference even if it were not perceived or measured (see Collier, 2008 for an application of this distinction in biology). Basically, substantive information is distinguished by its causal powers independently of whether these are observed. I will argue that there are various kinds of substantive information to be found in various sciences, and that they do indeed differ substantially from each other (pun intended). However they are related to each other such that each kind is a causal restriction on a more general kind, so that they form a succession, or hierarchy of restrictions. What they share, however, is that each kind of information makes a causal difference at its own level (and by inheritance to the preceding levels), but not necessarily to successive levels. So each bit of information at a given level makes at least a potential causal difference at this level. The explanation for how this level is formed, though, involves the preceding levels (usually just the first preced- 
ing) through cohesive processes at the lower level that lead to the unification of the higher level. So we get unification of kinds of substantive information by common principles applying at each level, but also by common causal processes across levels at each level that allow level formation. Although I believe that the unification of the sciences best follows this level oriented approach, rather than through reduction, I will not argue that general point here.

\section{Kinds of substantive information}

Information can be used as a tool in various ways, for example to estimate channel bandwidth of sensory pathways and various internal processes, the diversity of the genome and many others. In 1987 I searched the past three years of Biological Abstracts and found only seven out of several hundred that I judged to use the information concept substantively. The others either used the concept gratuitously (it was completely unnecessary) or used it as a measurement only. In order to determine substantive use in science it is important to judge whether the concept has referred to information in the world rather than just as a tool for measurement, or in some sort of metaphorical sense that can be eliminated.

\subsection{It from bit}

The most liberal and inclusive view is the "It from bit" view. It has originated independently from so many people that it is pointless to attribute an origin, though it probably goes back to Leibniz' view that the world has a logical structure of perceptions based in the ability to discriminate. The term is due to John Wheeler (1990), supported by Murray Gell-Mann (1995), and the view has recently been powerfully if controversially championed by Stephen Wolfram (2002). On this view, any causally (dynamically) grounded distinction makes a difference, thereby ensuring that it is information (MacKay, 1969, see also Bateson, 1973). On this view information is objective, and there is nothing else.

Although the idea applies to classical physics, its motivation comes primarily from quantum mechanics (QM). Each interaction in QM involves a sort of choice, and the choice can be represented in terms of bits. The equations governing QM, therefore, can be represented in terms of bits transferred. In QM the wave function is conserved (unitarity), so information is conserved (see Collier, 1999a; 2010a for more general way to apply the notion of information transfer to causation that does not imply preservation of information). This presents a real problem for the physics of "black holes", which seem to destroy information. Stephen Hawking has spent a long time considering this paradox, which is not yet completely resolved (he reversed his original position that information is lost irretrievably). The importance to the present paper is that it cannot be stated properly without invoking the concept of information. Interestingly, the black hole paradox is intimately connected to thermodynamics.

\subsubsection{Where does information come from?}

Physical information is an asymmetry in a system (Muller, 2007). This follows from the principle of indifference, according to which if there is nothing to distinguish two states, then they are effectively the same. Muller argues that, contrary to widespread thinking (e.g. Edwin Jaynes, who used a subjective principle of indifference grounded in Bayesian approaches to the definition of probability), it is possible in principle to determine the intrinsic information in a system in terms of its asymmetries. His work is based on some of my work arguing that information originates in symmetry breaking (Collier, 1996). In particular, nonequilibrium processes can produce symmetry breaking through splitting in phase changes, leading to greater diversity and the production of new cohesive structures (Collier, 2010b). I will have more to say about this in what follows, but it is the basis of cosmogenesis (Layzer, 1990) and the origin of much of the diversity we see in biology (Brooks \& Wiley, 1988). It is also the origin of new hierarchical levels of information in many kinds of systems (see section 1.3 below). 


\subsection{Negentropy}

After the development of the statistical ground of thermodynamics (which was not fully accepted until Einstein's explanation of Brownian motion in 1905), James Clark Maxwell (1871) investigated the nature of the Second Law, which says that entropy cannot decrease. To do this he invoked a demon residing in a box that could locate fast molecules and let them go through a gate into another box originally in thermodynamic equilibrium with the box the demon was in. If the demon could do this, then it could reduce the entropy of the two-box system. He soundly concluded from the demon thought experiment that the Second Law was statistical in nature (see Collier, 1990a for an explanation of his argument and some further consequences of the demon thought experiment).

The interesting aspect of the thought experiment from the perspective of this paper is that to do its job the demon must be able to obtain information about the state of the system without increasing the net entropy of the demon/box system. This cannot be done without creating information out of nothing. This violates a basic intuition about causation that it must involve resources that already exist (see Collier, 1990a for the full argument; the intuition is also used in Collier, 1999a). The argument does not show that the Second Law is necessary, but it does show that a) it is statistical, and b) that the entropy rate of a system cannot be intentionally reversed. The issue has led to a great deal of work on the physics of computation and the relation between physics and computation (for references see Collier, 1990a; 1999a; 2010a).

Leon Brillouin (1962) conjectured that information is equivalent to the complement of entropy, or negentropy, though the idea goes back to Erwin Schrödinger (1945). Brillouin clearly connected the idea to measurement, as in Maxwell's thought experiment, but also suggested that it was a physical value. This is how it is used in studies of the physics of computation. The basic idea here is that if we take the actual entropy of a system, $\mathrm{H}_{\text {Act }}$, and imagine all of the internal constraints released, the state of the "relaxed" system has the entropy $\mathrm{H}_{\text {MAX, }}$, the equilibrium entropy (note that this is not typically the entropy of the system if it is allowed to progress to equilibrium as an open system). The basic ideas are spelled out in (Collier; 1986; see also Collier, 1990b; Collier \& Hooker, 1999), though they can also be found in (Wiley \& Brooks, 1981), Landsberg (1984) and writings of a number of physicists. The importance of the idea is that in an expanding phase space both entropy and order (negentropy) can increase together. Furthermore, the process is able to drive the formation of even more order and organization, producing hierarchically organized systems (Layzer, 1990).

\subsection{Hierachical Information}

Donald MacKay (1965) asked the question of the nature of information in hierarchies, which he thought was fundamental to understanding organized information. This basic idea has been developed in a number of ways, including attempts to get an informational measure of organization (Bennett, 1985). However in the spirit of this paper, I am more interested in how information hierarchies might exist in nature.

There are two types of hierarchies found in nature. One is abstract, and refers in general. For example we have the physical, the chemical, the biological, the psychological and the social. These are not entities, and they have no direct causal powers - they don't do anything. On the other hand we can talk of the atoms that make up a particular molecule, the molecules that make up a particular cell, the cells that make up a particular body, and the bodies that make up a particular social group and a particular ecology (the former is part of the latter). These are particular entities and they have causal powers. They do things.

It is widely agreed in taxonomy that species are real entities (though some would take the real entities to be smaller groups like populations), whereas higher taxa are not. This does not mean that the higher taxa are fictional, however. We can't just classify species into higher taxa any way we please. They form what Duhem (1954) called a natural classification, the aim of scientific theory. However, if we are interested in substantive information, information that plays a role in what a thing is and does, we need to restrict ourselves to particular things. 
I have argued (Collier, 2003) that hierarchical information in particular systems must be arranged such that the levels in the hierarchy are cohesive. That is the levels are distinguished by causal closure that makes the level an entity. Basically, cohesion is a relation among the parts of something that make the parts parts of the same thing. So cells, for example, are distinguished by the organizational processes and chemical forces that make the cell more contained in a centripetal than a centrifugal direction (Ulanowicz, 1997). When we examine the closure of these processes and forces, the balances is inwards, holding the cell together and maintaining its existence. So the cell is at a higher, or more inclusive, than chemical processes and their organization.

\subsubsection{Cohesion}

Cohesion is the dynamical basis of individuation. It is both what gives things their identity, and what makes them different from other things (Collier, 2010b). Cohesion is not absolute, but comes in degrees, so there will be borderline cases. Furthermore, cohesion does not have just one dimension, but many, with the resulting cohesion being a sum of the many aspects. In general, systems, objects and properties will show a mixture of cohesive and noncohesive properties - not all the properties of something contribute to its cohesion, just the properties that keep it together. Cohesion is not merely the presence of interactions; the relevant interactions must be stabilizing for the thing of interest. Lastly, cohesive forces and processes need not be purely internal, but may involve external processes and forces that contribute to the closure of cohesion.

\subsubsection{Informational hierarchies}

In biological organisms the DNA is a code that can be transcribed and processed to produce both internal and external traits of the organism. To do this the information is transmitted through channels ${ }^{1}$ in which it is modified as it passes from one form to another until it is expressed in what we typically call traits (Collier, 2008). The information in the trait will contain information in the DNA, but it may also contain more information that came from other sources, or was produced by symmetry breaking during development. The whole organism is selected (by surviving to pass on its genes - and perhaps other forms of information as well - or not). This creates a feedback between environmental and genetic information that shapes the genetic information. Seldom is there any direct selection for genes (thought it happens).

Brooks and Wiley (1988) argued that there are several levels of information relevant to biological evolution. There is a genetic level, an organismal level and a population or species level (there may be further significant levels between these, but their approach is painted with broad strokes). Not all genetic information is expressed at the organism (phenotypic) level, and not all phenotypic information is expressed at the species level. However each of these levels is closed in each case by cohesive forces and processes. So we have a real and concrete informational hierarchy, assuming that the case can be made that the information at each level is substantive. I will return to this issue in section 2.2 below.

\subsection{Functional information}

In general a function is a property (more accurately a process) that has some end. The weakest form of function is what is called teleonomic: the end is implicit in the organization of whatever performs the function, and it leads to the maintenance of the function. Some functions are teleological, which requires some sort of representation of the end that is generated internally, and thus has intentionality. I will return to these later.

Biology is characterized by teleonomic processes that focus on preservation and reproduction. There are two basic approaches to biological function, the etiological and the organizational (Collier, 2004). The etiological approach defines the function of a trait in terms of what it was selected

\footnotetext{
${ }^{1}$ Throughout I use 'channel' in precise sense of Barwise and Seligman (1997) that I explain in some detail in (Collier 2008).
} 
for (being selected for means that the trait was selected because of what it does - just being selected can be accidental, e.g., if the trait is connected to another trait that is selected for). The heart, for example has the function of pumping blood, since that is what it was (and is) selected for. Calming babies by holding them over the heart is not the function of the heart because it was not selected for this calming effect. The effect is an accident. There are a number of problems with the etiological approach that I won't go into here, but one aspect of the account is that it implies that there is a specific "proper" function for each trait. The other main account of function is to define function in terms of organizational role. Unlike the etiological approach, a trait is functional not on its own, but together with other traits. We would say that it has functionality rather than a proper function. So the role of the heart is its contribution to the circulation of blood, which in turns contributes to nutrition and oxidation, which in turn contributes to survival and reproduction. The heart is functional just to the extent that its role is to contribute ultimately to survival and reproduction.

There is a clear connection between these two accounts. A trait that is functional in the organizational sense will make it more likely that an organism that has it will survive, all other things being equal. However the etiological account requires actual survival and reproduction. Traits that contribute to survival but aren't quite good enough are not functional. This is workable, but perhaps a bit odd. There is also the fact that a trait has to work in some sense in order for it to be selected for. The etiological approach seems to put the selective cart before the functional horse. Nonetheless, looking for traits that are selected for is a good strategy for finding functional traits. The ones that aren't good enough are probably long gone.

\subsubsection{Autonomy}

In order to understand organizational role better, the notion of autonomy is useful. In biological systems autonomy is self-regulation that leads overall to a greater likelihood of survival and reproduction. In fact it is a form of cohesion, but rather than being based mostly on forces (such as the cohesion of rocks and motorcars), it is based on organizational forces that typically involve rather small energy wells, and so, relatively small forces. The reason for this is that autonomy in an unpredictable or even changing environment requires shifting from one strategy to another, and switching is easier if it doesn't require a lot of force. Autonomy, then, is the organizational form of cohesion that leads to the persistence of the entity that has it (Collier, 1999b; 2002). Like all forms of cohesion, autonomy involves closure (Collier, 2000), and comes in degrees. The closure in this case is primarily the closure of processes. A trait is functional inasmuch as it contributes to autonomy. If we are speaking of an organism, then it is what leads to survival. If we are talking of a genetic lineage, then it is what promotes reproduction. Notice that these two ends can and often do compete with each other. This is typical of hierarchical or nested autonomy. Tragic perhaps, but more or less inevitable.

\subsubsection{Functional information}

Why would we think that function involves information in any way? First of all, traits are an expression of genetic information that are exposed to selective forces, so they fit the information model of section 1.3. However we are now in section 1.4 dealing with function, and unless we are to reduce functional information to hierarchical information, there must be some special role for information in at least some functions.

Marcello Barbieri (2001) has argued that much of biological functioning involves codes. The genetic code, no matter how important, is just one of these. The notion of a code seems to presuppose a notion of information: a code is a transformation from "one world to another" as Barbieri puts it, in which the encoded form acts as a stand-in for what it encodes. That is, there has to be an information channel between the two. So a code embodies an information channel, and we can employ information theoretic concepts to understand what it is that the coded processes do.

Whereas Barbieri thinks that codes are enough to understand many complex life processes, others think that a code alone is not enough. Various biosemioticians (too many to list here) think 
that these codes must be interpreted as well as being some form of mechanistic transcription. Fortunately, interesting though the issue is, it does not need to be resolved in order to justify the claim that at least some functionality involves substantive information. A somewhat worrying aspect of the interpretation requirement, though, is that it is often stated in terms of meaning. Indeed, interpretation itself strongly suggests meaning. However, like Barbieri, I prefer to keep meaning reserved for intentional systems, although I agree with the majority of biosemioticians that something is lacking in the code account alone.

\subsection{Intentionality and representation}

This section will be brief, since it is obvious to most people that our inner representations are meaningful and contain information, perhaps even about something that does not exist at all. In the case of codes, if there is a misrepresentation the code is malfunctioning, but this is not so for intentional representations. This suggests strongly that intentionality is something over and above the biological processes that support it.

Intentionality is clearly functional, so it inherits informational aspects of functionality. I have argued (Collier, 1999c) that intentional representations must have a certain degree of autonomy in order to serve their function.

... the main distinguishing feature of genuine semiosis appears to be the existence of a complex of anticipations related to other such complexes within which an interpretant can be formed. Representations permit complex anticipations and relations to other representations so as to allow intentional activity. Causal relations may look representational when they causally carry a signal about something, but this is not enough for them to be representational (Collier, 1999a, p. 128).

In other words, intentional representations must be integrated in to their context. Furthermore, it is this integration that gives them their meaning, not the underlying processes. So the cohesion of meaningful ideas is based in their integration with other ideas, as well as sensory inputs and motor outputs constrained by their flexibility in being applied as a unit in other contexts. The latter is required for them to be distinguishable from their contexts.

\subsection{Linguistic information}

The case that language involves substantive information is so obvious it hardly seems worth mentioning. It is worth noting, though, that language is necessarily social, and takes us into the social realm. It is more than just symbolic thought (pace Terrance Deacon), though symbolic thought is necessary for language. I would argue that all social activity that cannot be understood at the purely biological level (1.4) must be in a linguistic content to make sense, but establishing that is not necessary for justifying the substantive role of information in language. A more interesting question is whether the role of information in language can be reduced to the resources required for intentionality and representation. This is a thorny issue that I will not try to resolve here.

\section{Relations between the kinds of information}

Broadly, the kinds of information I have discussed above form an abstract hierarchy: fundamental physics, thermodynamics, hierarchical thermodynamic systems, functional systems, and intentional systems. Each type is nested within the previous one. Intentional systems are a type of functional system, which in turn are a type of hierarchical thermodynamic system. The latter are thermodynamic systems, which are physical systems. I will justify these claims in the subsection 2.1.

Furthermore, each successive kind is not just a restriction of the previous kind, but it is formed out of that kind by similar self-organizing processes, like those involved in Layzer's cosmogenesis (Layzer, 1990), Brooks and Wiley's unified approach to biology (Brooks \& Wiley, 1988), and Kauffman's origins of order (Kauffman, 1985; 1991; 1993). These processes produce new levels not just in the abstract, but in real and particular systems. I will argue this in section 2.2. 


\subsection{Nesting}

Stan Salthe $(1985 ; 1993)$ has argued that hierarchies involve a series of focal levels with each interacting primarily if not exclusively with the next and preceding levels. If my account in section $\mathbf{1}$ is coherent and basically correct, then information levels, both within the broad levels I describe as well as between them, are good candidates for this sort of hierarchical arrangement. In particular entities, there is a relationship of containment, corresponding to what Salthe calls a scalar hierarchy. Salthe also picks out a specification hierarchy, which is a relation between kinds such that each is contained within another, as in taxonomic systems. As I pointed out above, this sort of hierarchy is not necessarily substantive, though it may be natural. I once

suggested to Stan that the interesting cases come when the two hierarchies correspond, and I still believe this to be the case. Inasmuch as substantive information and its relations between kinds is "interesting", there should be a nesting that involves both specification and scale. Scalar nesting is guaranteed if each level in a system is contained physically and temporally within another higher level. If cohesion occurs at each level, then scalar nesting is guaranteed. Explicit recognition of the significance of cohesion is missing from Salthe's early accounts, though it was a central part of my account of informational hierarchies from the beginning (Collier, 1986).

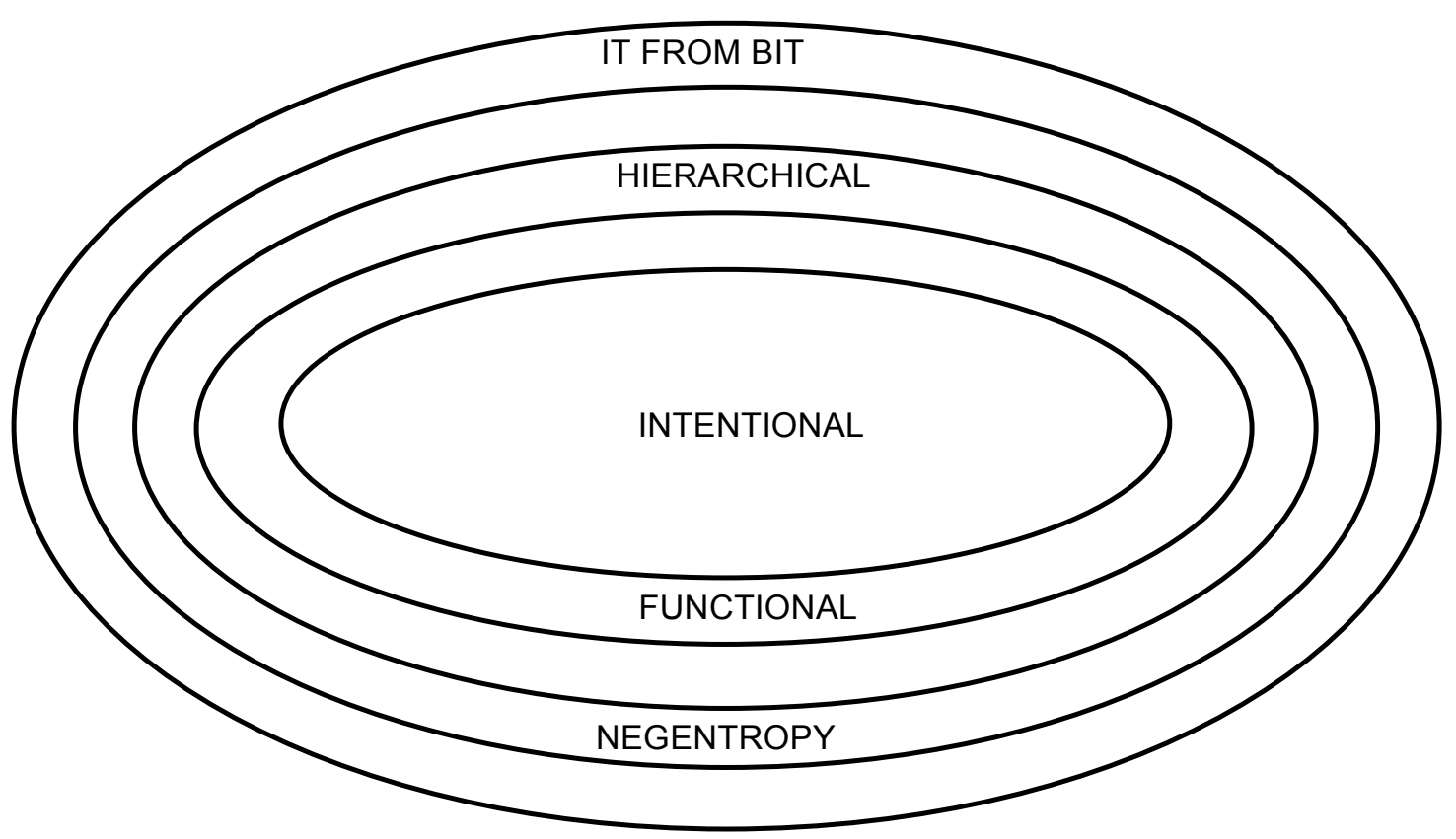

Figure 1: Nesting of major kinds of information (Collier, 2008)

To complete the picture it is necessary to show that each level in particular systems involves a cohesive constraint on the above level. It is fairly obvious that negentropy is nested within basic physics. I will defer its cohesive nature to section 2.2. Hierarchical information is nested within thermodynamic information (Collier, 2003). In the case of code based functionality at least, information is a restriction on hierarchical information (only some hierarchical information is functional). Furthermore, functionality occurs only under the far from equilibrium conditions that allow organization based autonomy. The move from functionality to intentionality, however, presents some difficulties. In order to make the case for nesting, intentional information must be a restriction on coding based functionality. This is not obvious. I will address this again in the conclusion of this paper. 


\subsection{Cohesion formation}

Each level is a restriction on the level preceding it (outside it in Figure 1). This restriction is created by the formation of cohesion through self-organization within the preceding (outer) level. For example, negentropy arises in the early universe as matter and energy go out of equilibrium as the universe expands. Prior to that there are merely local concentrations and dispersions of matter, but no steady separation. As the universe expands to the point that the expansion rate is greater than the equilibration rate matter condenses out, and the local entropy $\mathrm{H}_{\mathrm{ACT}}$ is lower than $\mathrm{H}_{\text {MAX }}$, creating local negentropy. Further hierarchical levels are formed when subsystems grow or expand, permitting bifurcations that produce new cohesive levels.

It is generally agreed that living systems formed through bifurcations within chemical systems, followed by selection. The formation of codes may have followed basic metabolism, or have come first, but stable living systems (lineages in this case) had to wait for the sort of reliable transmission provided by a code. So it is reasonable to argue that stable living things emerged out of dissipative processes feeding on negentropy (Schrödinger, 1945) through the stabilization of codes, a new form of information. It should be recalled that each of these new levels inherits the information of its predecessors, and that functional levels, for example, are subject to it from bit, negentropic and hierarchical dissipative processes within their own processes at their own level.

As mentioned above, however, the transition from functional processes to intentional processes is not very clear. I have noted that intentional processes rely on some degree of autonomy of those processes, inheriting characteristics of functionality, but at a new level. What more is required for intentionality to emerge will have to wait for further work, though I have made a suggestion in my conclusions.

\section{Conclusions}

For each kind of substantive information used in the sciences there is a distinct level formed by bifurcations that form cohesive structures at the next higher level. This is reflected in the information at each level, which inherits the properties of the lower level, but produces new asymmetries at its own level through the formation of new cohesions peculiar to the level. The only really problematic transition with current knowledge is from the biological to the intentional level. This is because it is not obvious that the information in the intentional level is based on biological codes that make up at least part of the functional level (see Barbieri, 2010 for a possible approach). A possible solution is a better understanding of the nature of neural transmission and the nature of neural transmitters, which may work together like a code. It seems obvious that the functioning of the neural system depends on the transmission of substantive information. Perhaps there is another level between the functional and the intentional - the nervous system. This would make sense in the overall picture, since the nervous system differentiated from the rest of the body both evolutionarily and developmentally. This in itself is not enough because other organs have also differentiated, but do not constitute a separate information system. (The immune system might be a notable exception. It might also have its own cohesion and informational properties (Matthen \& Levy, 1984)) It would be required to show that the nervous system was autonomous to some degree prior to intentionality (which presumably emerged out of it). Alternatively, it might be possible to show that intentionality can be fully reduced to nervous system activity, but I doubt this.

\section{References}

Barbieri, M. (2001). The Organic Codes: The Birth of Semantic Biology. Acona, Italy: peQuod. [Reprinted in 2002 by Cambridge University Press].

Barbieri. M. (2010). On the Origin of Language: A Bridge Between Biolinguistics and Biosemiotics. Biosemiotics, 3, 201-223. Barwise, J. \& J. Seligman (1997). Information Flow: The Logic of Distributed Systems. Cambridge: University of Cambridge Press. 
Bateson, G. (1973). Steps to an ecology of mind. London: Paladin.

Bennett C.H. (1985). Dissipation, information, computational complexity and the definition of organization. In D. Pines (Ed.), Emerging Syntheses In Science: Proceedings of the Founding Workshops of the Santa Fe Institute. Redwood City, CA: Addison West Publishing Company.

Brooks, D.R. \& E.O. Wiley. (1988). Evolution as entropy: Toward a unified theory of biology. Chicago: University of Chicago Press.

Collier, J (1986). Entropy in evolution. Biology and Philosophy, 1, 5-24.

Collier, J. (1990a). Two faces of Maxwell's demon reveal the nature of irreversibility. Studies in the History and Philosophy of Science, 21, 257-268.

Collier, J. (1990b). Intrinsic information. In P. P. Hanson (Ed.), Information, language and cognition. Oxford: Oxford University Press.

Collier, John (1996). Information originates in symmetry breaking. Symmetry: Culture \& Science, 7, 247-256.

Collier, J. (1999a). Causation is the transfer of information. In H. Sankey (Ed.), Causation, natural laws and explanation (pp. 279-331). Dordrecht: Kluwer.

Collier, J. (199b). Autonomy in anticipatory systems: Significance for functionality, intentionality and meaning. In: D.M., Dubois (Ed.), Proceedings of the American Institute of Physics from CASYS '98 - Second International Conference on Computing anticipatory systems (pp. 75-81). Woodbury, NY.

Collier, J. (199c). The dynamical basis of information and the origins of semiosis. In E. Taborsky (Ed.), Semiosis. Evolution. Energy towards a reconceptualization of the sign (pp. 111-136). Aachen: Shaker Verlag.

Collier, J. (2000) Autonomy and process closure as the basis for functionality. In J. L. R. Chandler \& G. van de Vijver (Eds.), Closure: Emergent organizations and their dynamics. Annals of the New York Academy of Science, 901: 280-291.

Collier, J. (2002). What is autonomy? Partial proceedings of CASYS '01: Fifth International Conference on Computing Anticipatory Systems, International Journal of Computing Anticipatory Systems, 12, 212-221.

Collier, J. (2003). Hierarchical dynamical information systems with a focus on biology. Entropy, 5, 100-124.

Collier, J. (2004) Interactively open autonomy unifies two approaches to function. In D. M. Dubois (Ed.), Proceedings of the American Institute of Physics from CASYS '03 - Sixth International Conference on Computing anticipatory systems (pp. 228-235). Melville, NY.

Collier, J. (2008). Information in biological systems. In P. Adriaans \& J. van Benthem (Eds.), Handbook of philosophy of science, Volume 8: Philosophy of information (pp 763-787). Dordrecht: North-Holland.

Collier, J. (2010a). Information, causation and computation. In G.D. Crnkovic \& M. Burgin (Eds.), Information and computation: Essays on scientific and philosophical understanding of foundations of information and computation (World Scientific Series in Information Studies). Singapore: World Scientific 2010.

Collier, J. (2010b). A dynamical approach to identity and diversity in complex systems. In P. Cilliers \& R. Preiser (Eds.), Complexity, difference and identity: An ethical perspective (pp. 83-97). Berlin: Springer.

Collier, J. \& Hooker, C.A. (1999). Complexly organised dynamical systems. Open Systems and Information Dynamics, 6 , 241-302.

Duhem, P. (1954) The aim and structure of physical theory. Princeton: Princeton University Press.

Giddens, A. (1984). The constitution of society. Berkeley: University of California Press.

Gell-Mann, M. (1995). The quark and the jaguar: Adventures in the simple and the complex. New York: Owl Books.

Kauffman, S. A. (1985). Self-organisation, selective adaptation, and its limits. In Depew \& Weber (Eds.), Evolution at a crossroads. Cambridge, MA: MIT Press.

Kauffman, S., A., (1991). Antichaos and adaptation. Scientific American, 265, 64-70.

Kauffman, S. A. (1993). Origins of order: Self-organisation and selection in evolution. New York: Oxford University Press.

Landsberg, P.T. (1984). Can entropy and "order" increase together? Physics Letters, 102A, 171-173.

Layzer, D. (1990). Cosmogenesis: The growth of order in the universe. New York: Oxford University Press.

MacKay, D. M. (1969). Information, mechanism and meaning. Cambridge, MA: MIT Press.

Matthen, M. \& E. Levy (1984). Teleology, error, and the human immune system. Journal of Philosophy, 81(7), 351-372.

Maxwell, J.C. (1871). Theory of heat. London: Longmans, Green and Co.

Muller, S. J. (2007). Asymmetry: The Foundation of information. Berlin: Springer.

Salthe, S.N. (1985). Evolving hierarchical systems. New York: Columbia University Press.

Salthe, S.N. (1993). Development and evolution: Complexity and change in biology. Cambridge, MA: MIT Press.

Schrödinger, E. (1945), What is life? Cambridge: Cambridge University Press.

Ulanowicz, R.E. (1997). Ecology: The ascendant perspective. New York: Columbia University Press.

Wheeler, J.A. (1990). Information, physics, quantum: The Search for links. In W. Zurek (Ed.), Complexity, entropy, and the physics of information. Redwood City, CA: Addison-Wesley.

Wiley, E.O. \& Brooks, D.R. (1981). Victims of history: A nonequilibrium approach to evolution. Systematic Zoology, 31, 1-24. Wolfram, S. (2002). A new kind of science. Champaign, IL: Wolfram Media. 


\section{About the Author}

\section{John Collier}

John Collier was educated at MIT, UCLA and Western Ontario in Planetary Science and Philosophy. For a number of years he went back and forth between the two until he settled into Philosophy. He has had lecturing and/or research positions in five countries and four continents, but now seems to have settled in Durban, South Africa, where he has now lived longer than he has lived anyplace else in his life. His current interests are metaphysics, complexity, information theory, evolutionary ethics and the way they overlap. His doctoral dissertation was on meaning and reference in theory change. 